\title{
ON THE NOTION OF SUMMABILITY FOR THE LIMIT OF A
}

\section{FUNCTION OF A CONTINUOUS VARIABLE*}

BY

\section{L. SILVERMAN \\ 1. INTRODUCTION†}

A sequence of numbers $u(m)$ may be regarded as a function of a discrete variable $m$. To generalize the notion of the limit of this sequence, a new sequence $v(m)$ is defined by means of the transformation

$$
v(m)=\sum_{n=1}^{m} k(m, n) u(n),
$$

where $k(m, n)$ is a function of two discrete variables. This transformation is regular if the existence of

implies the existence of

$$
\lim _{m=\infty} u(m)
$$

$$
\lim _{m=\infty} v(m)
$$

and the equality of the two limits. Examples of regular transformations are those of Cesàro and Hölder, of which the following are simple cases:

$$
\begin{gathered}
v_{1}^{\prime}(m)=v_{1}^{\prime \prime}(m)=\frac{1}{m} \sum_{n=1}^{m} u(n), \quad v_{2}^{\prime}(m)=\frac{\sum_{n=1}^{m} \sum_{p=1}^{n} u(p)}{\sum_{n=1}^{m} n}, \\
v_{2}^{\prime \prime}(m)=\frac{1}{m} \sum_{n=1}^{m} v_{1}^{\prime \prime}(n),
\end{gathered}
$$

where the superscripts refer respectively to the definitions of Cesàro and' Hölder and the subscripts to the order of summability. A necessary and sufficient condition that a transformation be regular is:

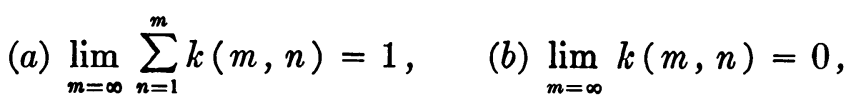

$$
\text { (c) } \sum_{n=1}^{m}|k(m, n)|<A \text {. }
$$

\footnotetext{
* Presented to the Society, January 1, 1915.

$\dagger$ I wish to express my indebtedness to Professor W. A. Hurwitz for a number of suggestions. ‡ Toeplitz, Prace matemat y c z no-f i y c z no, vol. 22 (1911), p. 113.
} 
It is natural to replace the discrete variable $m$ by the continuous variable* $x$, $u(m)$ by $u(x)$, the auxiliary function $k(m, n)$ by $k(x, y)$, a function of two continuous variables, the sign of summation by an integral sign, and then to study the transformation

$$
v(x)=\int_{a}^{x} k(x, y) u(y) d y
$$

where $u(x)$ is bounded and integrable, $a \leqq x \leqq x_{1}$, and $k(x, y)$ is integrable in $y$ for each $x, a \leqq y \leqq x$. The function $k(x, y)$ is the kernel of the transformation. The transformation is regular if $\lim _{x=\infty} u(x)$ implies the existence of $\lim _{x=\infty} v(x)$ and the equality of the two limits. Corresponding to the examples (1) we have, taking for convenience $a=0$, the transformations: $\dagger$

$$
\begin{gathered}
v_{1}^{\prime}(x)=v_{1}^{\prime \prime}(x)=\frac{1}{x} \int_{0}^{x} u(y) d y, \quad v_{2}^{\prime}(x)=\frac{\int_{0}^{x} \int_{0}^{y} u(s) d s d y}{\int_{0}^{x} y d y}, \\
v_{2}^{\prime \prime}(x)=\frac{1}{x} \int_{0}^{x} v_{1}^{\prime \prime}(y) d y .
\end{gathered}
$$

Changing the order of integration in the numerator of the expression for $v_{2}^{\prime}(x)$ and integrating once, we have

$$
v_{2}^{\prime}(x)=\frac{2}{x^{2}} \int_{0}^{x}(x-y) u(y) d y
$$

so that the kernels for the transformations of Cesàroł of orders 1 and 2 are respectively

$$
k_{1}^{\prime}(x, y)=\frac{1}{x}, \quad k_{2}^{\prime}(x, y)=\frac{2(x-y)}{x^{2}}
$$

Following the case of series we are led to inquire whether conditions similar to (2) constitute a necessary and sufficient condition for the regularity of the transformation (3). In answer to this question we have the theorems which follow in the next section.

* The first to carry out this idea to extend Cesàro's and Hölder's definitions of summability of series to the case of integrals was Du Bois-Reymond, J o u r n a l f ür Mathem a ti k, vol. 100 (1887), p. 354.

† See Landau, Die Identität des Cesdroschen und Hölderschen Grenzwertes für Integrale, Sä ch s is che Bericht e, vol. 65 (1913), p. 131.

$\ddagger$ That is, the transformations in the case of a continuous variable corresponding to those of Cesaro for the case of sequences. 


\section{Conditions for REgularity}

Theorem I. Let $k(x, y)$ be defined, $a<x, a \leqq y \leqq x$, and integrable in $y$ for each $x$; then a sufficient condition that $k(x, y)$ correspond to a regular transformation is:

$$
\begin{aligned}
& \text { (a) } \lim _{x=\infty} \int_{a}^{x} k(x, y) d y=1, \\
& \text { (b) } \lim _{x=\infty} k(x, y)=0 \text { uniformly in } y, \quad a \leqq y \leqq q, \\
& \text { (c) } \int_{a}^{x}|k(x, y)| d y<A, \quad a<x,
\end{aligned}
$$

where $q$ is an arbitrary constant and $A$ is a positive constant.

We wish to prove that for any bounded and integrable function $u(x)$ the existence of $\lim _{x=\infty} u(x)$ implies the existence of

$$
\lim _{x=\infty} \int_{a}^{x} k(x, s) u(s) d s
$$

and the equality of the two limits.

Let $\lim _{x=\infty} u(x)=l$; then by $(a)$ we may write,

$$
l=l \int_{a}^{x} k(x, s) d s+\eta(x),
$$

where $\lim _{x=\infty} \eta(x)=0$; so that

$$
\int_{a}^{x} k(x, s) u(s) d s-l=\int_{a}^{x} k(x, s)[u(s)-l] d s-\eta(x) .
$$

Choosing $p$ so large that $|u(x)-l|<\epsilon, x \geqq p$, then holding $p$ fixed and denoting by $M$ a number greater than $|u(x)-l|$ in the interval $a \leqq x \leqq p$, we have for $x>p$,

$$
\begin{aligned}
&\left|\int_{a}^{x} k(x, s) u(s) d s-l\right| \leqq \int_{a}^{p}|k(x, s)||u(s)-l| d s \\
&+\int_{p}^{x}|k(x, s)||u(s)-l| d s+|\eta(x)| \\
& \quad \leqq M \int_{a}^{p}|k(x, s)| d s+\epsilon \int_{p}^{x}|k(x, s)| d s+|\eta(x)|
\end{aligned}
$$

Since by $(c)$

and by (b)

$$
\int_{p}^{x}|k(x, s)| d s \leqq \int_{a}^{x}|k(x, s)| d s<A
$$

$$
\int_{a}^{p}|k(x, s)| d s<\frac{\epsilon}{M(p-a)} \int_{a}^{p} d s=-\frac{\epsilon}{M}, \quad x>X,
$$


it follows that, if $X^{\prime}$ is the greater of the numbers $p$ and $X$,

so that

$$
\left|\int_{a}^{x} k(x, s) u(s) d s-l\right|<\epsilon+\epsilon A+|\eta(x)|, \quad x>X^{\prime},
$$

$$
\lim _{x=\infty} \sup _{x}\left|\int_{a}^{x} k(x, s) u(s) d s-l\right| \leqq \epsilon+\epsilon A .
$$

Since the superior limit is less than an arbitrarily small positive constant, the actual limit must be zero. Therefore,

$$
\lim _{x=\infty} \int_{a}^{x} k(x, s) u(s) d s=l
$$

Theorem II. Let $k(x, y)$ be defined, $a<x, a \leqq y \leqq x$, and continuous in $y$ uniformly with respect to $x, x>h>a$, and let the zeroes of $k(x, y)$ for each $x$ consist of a set of segments* and of a set $\dagger$ of points of measure zero; then a necessary and sufficient condition that $k(x, y)$ correspond to a regular transformation is that $k(x, y)$ satisfy (6).

That these conditions are sufficient has been proved in the preceding theorem; we proceed to show that they are necessary. We accordingly assume that whenever

then

$$
\lim _{x=\infty} u(x)=l,
$$

$$
\lim _{x=\infty} \int_{a}^{x} k(x, s) u(s) d s=l,
$$

and we prove that each of the conditions (6) follows as a necessary consequence.

(a) Consider the special case in which $u(x)=1, a \leqq x$, so that

then

$$
\lim _{x=\infty} u(x)=1
$$

$$
\lim _{x=\infty} \int_{a}^{x} k(x, s) d s=1 .
$$

(b) Consider the special case in which

so that $\lim _{x=\infty} u(x)=0$.

$$
\begin{aligned}
u(x) & =1, \quad a \leqq x \leqq y, \\
& =0, \quad y<x ;
\end{aligned}
$$

Letting

$$
\int_{a}^{y} k(x, s) d s=g(x, y),
$$

* The set of segments is necessarily countable. See Hobson, Theory of Functions of a Real Variable, p. 82.

$\dagger$ Either of the sets may be null.

Trans. Am. Math. Soc. 19 
we have

$$
\int_{a}^{x} k(x, s) u(s) d s=\int_{a}^{y} k(x, s) d s=g(x, y) ;
$$

so that $\lim _{x=\infty} g(x, y)=0$ for each $y$, and hence

Since

$$
\lim _{x=\infty} \frac{g(x, y+r)-g(x, y)}{r}=0 .
$$

$$
\lim _{r=0} \frac{g(x, y+r)-g(x, y)}{r}=\frac{\partial g(x, y)}{\partial y}=k(x, y),
$$

it will follow that

$$
\begin{aligned}
\lim _{x=\infty} k(x, y) & =\lim _{x=\infty} \lim _{r=0} \frac{g(x, y+r)-g(x, y)}{r} \\
& =\lim _{r=0} \lim _{x=\infty} \frac{g(x, y+r)-g(x, y)}{r}=0,
\end{aligned}
$$

if we show that for each constant $y$ the limit in (7) is approached uniformly for all $x \geqq h$. To show this, we write

$$
\frac{g(x, y+r)-g(x, y)}{r}=\frac{1}{r} \int_{y}^{y+r} k(x, s) d s=k\left(x, \bar{y}_{x}\right),
$$

where $\bar{y}_{x}$ lies between $y$ and $y+r$.

From the assumption regarding the uniform continuity of $k(x, y)$ when $y \geqq a$, we have

$$
\left|k\left(x, y_{1}\right)-k(x, y)\right|<\epsilon, \quad\left|y_{1}-y\right|<\delta, \quad x \geqq h,
$$

where $\epsilon$ is a given arbitrarily small positive constant and $\delta$ depends only upon $\epsilon$ and $h$. Thus for all $x \geqq h$ and for $|r|<\delta$, we have, since

$$
\left|y-\bar{y}_{x}\right|<|r|<\delta, \quad\left|k\left(x, \bar{y}_{x}\right)-k(x, y)\right|<\epsilon .
$$

The limit in (7) is accordingly uniform in $x$. It follows that the limit of $k(x, y)$ is zero for each $y$.

We shall now show that

$$
\lim _{x=\infty} k(x, y)=0 \text { uniformly in } y, \quad a \leqq y \leqq q,
$$

where $q$ is arbitrary. Suppose this limit exists non-uniformly in $y$ in some finite interval, $p_{1} \leqq y \leqq q_{1}$. Then the limit must exist non-uniformly in one of the half-intervals. Subdividing the half-intervals and proceeding in the usual way, we define a value $\xi$ of $y$ such that in every interval about $\xi$ the limit of $k(x, y)$ exists non-uniformly in $y$. We shall now obtain a contradiction by showing that in at least one interval about $\xi$, the limit of $k(x, y)$ exists uniformly in $y$. 
Since $k(x, y)$ is continuous in $y$ uniformly with respect to $x$, we have

$$
|k(x, \xi)-k(x, y)|<\frac{\epsilon}{2}, \quad|\xi-y|<\delta, \quad x \geqq h,
$$

and since the limit of $k(x, y)$ exists for each $y$, including $y=\xi$, we have

$$
|k(x, \xi)|<\frac{\epsilon}{2}, \quad x>X .
$$

Adding these inequalities, we obtain

$$
|k(x, y)|<\epsilon, \quad x>X^{\prime}, \quad|\xi-y|<\delta,
$$

where $X^{\prime}$ is the greater of the numbers $h$ and $X$. We have thus proved that the limit of $k(x, y)$ can exist non-uniformly in no finite interval; hence

$$
\lim _{x=\infty} k(x, y)=0 \text { uniformly in } y, \quad a \leqq y \leqq q .
$$

(c) We are now to prove that there exists a constant $A$ such that

$$
\phi(x)=\int_{a}^{x}|k(x, s)| d s<A, \quad a<x .
$$

Let us assume this assertion untrue; then for some $x_{1}, \phi\left(x_{1}\right)>10^{2}$. Considering the special case in which*

so that

$$
u(y)=\frac{1}{10} \operatorname{sgn} k\left(x_{1}, y\right), \quad a \leqq y \leqq x_{1},
$$

and letting

$$
u(y) k\left(x_{1}, y\right)=\frac{1}{10}\left|k\left(x_{1}, y\right)\right|,
$$

we have

$$
g(x)=\int_{a}^{x} k(x, s) u(s) d s,
$$

$$
g\left(x_{1}\right)=\frac{1}{10} \int_{a}^{x_{1}}\left|k\left(x_{1}, s\right)\right| d s=\frac{1}{10} \phi\left(x_{1}\right)>10 .
$$

Furthermore, from the uniform approach to zero of $k(x, y)$, proved in $(b)$, it follows that

$$
\lim _{x=\infty} \int_{a}^{x_{1}}|k(x, s)| d s=0
$$

so that

$$
\int_{a}^{x_{1}}|k(x, s)| d s<1, \quad x>X_{1}>x_{1} \text {. }
$$

Now choose $x_{2}>X_{1}$ so large that

$$
\phi\left(x_{2}\right)>10^{4}+10+1 .
$$

* The function sgn $z$ is defined, as usual, to have the value $|z| / z$ when $z \neq 0$, and the value 0 when $z=0$. 
The function $u(y)$ has been defined for the interval $a \leqq y \leqq x_{1}$; let us now extend the domain of definition to the interval $x_{1}<y \leqq x_{2}$ as follows:

Then

$$
u(y)=\frac{1}{10^{2}} \operatorname{sgn} k\left(x_{2}, y\right), \quad x_{1}<y \leqq x_{2} .
$$

$$
\begin{aligned}
g\left(x_{2}\right) & =\int_{a}^{x_{2}} k\left(x_{2}, s\right) u(s) d s \\
& =\int_{a}^{x_{1}} k\left(x_{2}, s\right) u(s) d s+\int_{x_{1}}^{x_{2}} k\left(x_{2}, s\right) u(s) d s \\
& =\int_{a}^{x_{1}} k\left(x_{2}, s\right) u(s) d s+\frac{1}{10^{2}} \int_{x_{1}}^{x_{2}}\left|k\left(x_{2}, s\right)\right| d s \\
& =\frac{1}{10^{2}} \int_{a}^{x_{2}}\left|k\left(x_{2}, s\right)\right| d s-\int_{a}^{x_{2}}\left[\frac{1}{10^{2}}\left|k\left(x_{2}, s\right)\right|-k\left(x_{2}, s\right) u(s)\right] d s .
\end{aligned}
$$

Thus

$$
\begin{aligned}
\left|g\left(x_{2}\right)\right| & \geqq \frac{1}{10^{2}} \phi\left(x_{2}\right)-\left|\int_{a}^{x_{1}}\left[\frac{1}{10^{2}}\left|k\left(x_{2}, s\right)\right|-k\left(x_{2}, s\right) u(s)\right] d s\right| \\
& \geqq \frac{1}{10^{2}} \phi\left(x_{2}\right)-\int_{a}^{x_{1}}\left[\frac{1}{10^{2}}\left|k\left(x_{2}, s\right)\right|+\left|k\left(x_{2}, s\right)\right||u(s)|\right] d s \\
& =\frac{1}{10^{2}} \phi\left(x_{2}\right)-\int_{a}^{x_{1}}\left(\frac{1}{10^{2}}+\frac{1}{10}\right)\left|k\left(x_{2}, s\right)\right| d s .
\end{aligned}
$$

From (8) and (9) it follows that

$$
g\left(x_{2}\right) \geqq \frac{10^{4}+10+1}{10^{2}}-\frac{1}{10^{2}}-\frac{1}{10}=10^{2} .
$$

Continuing in this way, we can define a sequence of numbers $x_{n}$ such that

$$
\lim _{n=\infty} x_{n}=\infty, \quad \lim _{n=\infty}\left|g\left(x_{n}\right)\right|=\infty ;
$$

therefore it is not true that the limit of $g(x)$ is zero.

On the other hand, from the hypothesis as to the roots of $k(x, y), u(y)$ is integrable,* and from its definition $u(y)$ is bounded; hence, since

$$
\lim _{x=\infty} u(x)=0,
$$

it follows from the fundamental property of regular transformations $(\S 1)$ that

* The end-points of the set of intervals mentioned in the theorem form a countable set (see first footnote, p. 287); the measure of the set of all the points of discontinuity of $u(y)$ is accordingly zero. Hence $u(y)$ is integrable. See Hobson, loc. cit., p. 342. 


$$
\lim _{x=\infty} g(x)=\lim _{x=\infty} \int_{a}^{x} k(x, s) u(s) d s=0 .
$$

The contradiction proves that

$$
\int_{a}^{x}|k(x, s)| d s<A, \quad a<x .
$$

\section{The CASE of INFINITE Kernel}

It will be seen that the kernels given in (5) of the transformations of Cesàro satisfy the conditions of the preceding theorem. To see whether also the kernel of the transformation* of Hölder of order 2 satisfies those conditions, let us find the expression for $k_{2}^{\prime \prime}(x, y)$. We have from (4), for $x>0$,

$$
\begin{aligned}
v_{2}^{\prime \prime}(x) & =\frac{1}{x} \int_{0}^{x} v_{1}^{\prime \prime}(y) d y=\frac{1}{x} \int_{0}^{x} \int_{0}^{y} \frac{1}{y} u(s) d s d y \\
& =\lim _{e=0} \frac{1}{x} \int_{e}^{x} \int_{0}^{y} \frac{1}{y} u(s) d s d y \\
& =\lim _{e=0} \frac{1}{x}\left[\log y \int_{0}^{y} u(s) d s\right]_{e}^{x}-\lim _{e=0} \frac{1}{x} \int_{e}^{x} u(y) \log y d y \\
& =\frac{1}{x} \int_{0}^{x} \log \left(\frac{x}{y}\right) u(y) d y,
\end{aligned}
$$

since

$$
\left|\log \epsilon \int_{0}^{\epsilon} u(s) d s\right|<M \epsilon|\log \epsilon|,
$$

where $M$, a constant independent of $\epsilon$, is an upper bound of $u(x)$. Thus

$$
k_{2}^{\prime \prime}(x, y)=\frac{1}{x} \log \frac{x}{y}
$$

This kernel does not satisfy the conditions of the preceding theorem, since it fails to be continuous for $y=0$. We are thus led to study the transformation

$$
v(x)=\int_{a}^{x} k(x, s) u(s) d s,
$$

where $k(x, y)$ may become infinite for $x=a$ while the integral converges; $\ddagger$ to define as regular any such transformation for which the existence of

$$
\lim _{x=\infty} u(x)
$$

* The kernel of Hölder's transformation of order 1 is identical with that of Cesaro of order 1. $\dagger$ It is here assumed that $u(x)$ is continuous; everywhere else in this paper $u(x)$ is assumed to be merely bounded and integrable.

$\ddagger$ As in $\& 1, u(x)$ is assumed to be bounded and integrable, $a \leqq x \leqq x_{1}$. 
implies the existence of

$$
\lim _{x=\infty} \int_{a}^{x} k(x, s) u(s) d s
$$

and the equality of the two limits; and to inquire whether the results of the preceding section can be extended to this class of transformations. It will be observed that the third condition of (6) will now necessitate the further assumption that

$$
\int_{a}^{x}|k(x, y)| d y
$$

converge. With the additional assumption that this integral converges uniformly in $x$, a theorem similar to Theorem I of the preceding section can be proved for the more general class of transformations under consideration.

Theorem III. Let $k(x, y)$ be defined, $a<y \leqq x$, and integrable in $y$ for each $x$; then a sufficient condition that $k(x, y)$ correspond to a regular transformation is:

$$
\int_{a}^{b}|k(x, y)| d y \text { converges, } \quad \lim _{x=\infty} \int_{a}^{b}|k(x, y)| d y=0,
$$

$$
\lim _{x=\infty} \int_{a}^{x} k(x, y) d y=1, \quad \int_{a}^{x}|k(x, y)| d y<A, \quad a<x,
$$

where $b$ is an arbitrary constant greater than $a$, and $A$ is a positive constant.

In order to prove this theorem it is necessary to bear in mind that the convergence of the integral ${ }^{*}$

$$
\int_{a}^{x}|k(x, y)| d y
$$

implies the convergence of the integral

$$
\int_{a}^{x}|k(x, y)||u(y)| d y
$$

since $u(y)$ is bounded. We may now follow the successive steps of the proof in Theorem I, observing that every integral involved is necessarily convergent.

We shall now state a necessary and sufficient condition that a kernel $k(x, y)$, for the more general class of transformations considered in this section, correspond to a regular transformation. In this theorem, however, a far greater restriction is put on the kernel than in the preceding ones; we assume, in fact,

* That is, the existence of the limit, as $h$ approaches zero, of the integral

$$
\int_{a+h}^{x}|k(x, y)| d y .
$$


that the kernel is positive. It should be pointed out that the kernels corresponding to the transformations of Cesàro and Hölder satisfy this condition.

Theorem IV. Let $k(x, y)$ be positive or zero, $a<y \leqq x$, and integrable in $y$ for each $x$; then a necessary and sufficient condition that $k(x, y)$ correspond to a regular transformation is

$$
\begin{gathered}
\int_{a}^{b} k(x, y) d y \text { converges; } \quad \lim _{x=\infty} \int_{a}^{b} k(x, y) d y=0 \\
\lim _{x=\infty} \int_{a}^{x} k(x, y) d y=1
\end{gathered}
$$

where $b$ is an arbitrary constant greater than $a$.

That the condition is sufficient follows immediately from Theorem III, if we make use of the hypothesis that $k(x, y)$ is positive; we accordingly proceed to the proof of the necessity of conditions (11). The hypothesis is that the convergence of $u(x)$ to the value $l$ implies, for every bounded and integrable function $u(x)$, the two consequences,

converges, and

$$
\int_{a}^{x} k(x, s) u(s) d s
$$

$$
\lim _{x=\infty} \int_{a}^{x} k(x, s) u(s) d s=l .
$$

To prove the first two conditions (11), consider the special case in which

$$
\begin{aligned}
u(y) & =1, & & y \leqq b, \\
& =0, & & y>b,
\end{aligned}
$$

so that $\lim _{x=\infty} u(x)=0$. Hence, since

$$
\int_{a}^{x} k(x, s) u(s) d s=\int_{a}^{b} k(x, s) d s,
$$

the integral on the right converges; which proves the first of conditions (11). Furthermore

$$
\lim _{x=\infty} \int_{a}^{x} k(x, s) u(s) d s=\lim _{x=\infty} \int_{a}^{b} k(x, s) d s=0 ;
$$

which proves the second of conditions (11). The third condition is proved as in Theorem II.

\section{Conclusion}

Three things may be said concerning the generalization for the limit of a function of a continuous variable as that variable becomes infinite. In the first place, improper integrals are included as a special case; for if we have 
under consideration

we may write

$$
\int_{a}^{\infty} \phi(x) d x
$$

$$
u(x)=\int_{a}^{x} \phi(y) d y
$$

and inquire as to the existence of

$$
\lim _{x=\infty} u(x) .
$$

Secondly, we need not restrict ourselves to the case where the independent variable approaches infinity; if we wish to study $\lim _{x=a} u(x)$, we may make the substitution

and study

$$
x=a+\frac{1}{t}
$$

$$
\lim _{t=\infty} u\left(a+\frac{1}{t}\right) \text {. }
$$

In particular we may generalize the notion of continuity. Finally, we wish to call attention to the fact that the class of transformations considered does not include the identity. We shall therefore study transformations of the form

$$
v(x)=\alpha u(x)+(1-\alpha) \int_{a}^{x} k(x, s) u(s) d s,
$$

which gives for $\alpha=0$ the transformations already considered, and for $\alpha=1$ the identity. It is clear that the conditions for regularity obtained in the preceding section apply to this case also; in fact, the statements and proofs of Theorems I-IV may be repeated word for word for this more general class of transformations.

In another paper we shall discuss the consistency and equivalence of these transformations.

Cornell UnIversity, August, 1915 